\title{
Análise e Implementação de Modelos de Turbulência $\kappa-\varepsilon$ para Simulação de Escoamentos Incompressíveis Envolvendo Superfícies Livres e Rígidas
}

V.G. FERREIRA, Departamento de Estatística, Matemática Aplicada e Computacional, Instituto de Geociências e Ciências Exatas, UNESP, 13500-230 Rio Claro, SP, Brasil

J.A. CUMinATO, M.F. TOMÉ, A.O. FORTUNA, N. MANGIAVACCHI, A.F. CASTELO, L.G. NONATO, Departamento de Ciências de Computação e Estatística, Instituto de Ciências Matemáticas e de Computação, USP, 13566-590 São Carlos, SP, Brasil.

\begin{abstract}
Resumo. O bom desempenho do algoritmo numérico é o pré-requisito essencial para a simulação de escoamentos em regime turbulento. O presente trabalho é destinado à discussão de resultados numéricos para escoamentos incompressíveis turbulentos na presena de superfícies livres e/ou rígidas. Os problemas considerados são o de um jato incidindo sobre uma superfície sólida e o problema do desprendimento de vórtices atrás de um obstáculo. O código GENSMAC, que emprega a formulação em variáveis primitivas e diferenças finitas para resolver as equações transientes de Navier-Stokes, foi usado no estudo. Dois modelos de turbulência tipo $\kappa-\varepsilon$ foram analisados e implementados: o modelo tradicional e um modelo para baixos números de Reynolds. Os termos convectivos das equações diferenciais parciais de transporte foram aproximados por esquemas de conveç̧ão limitados de segunda ordem. Os resultados obtidos confirmam a importncia de se tratar adequadamente os termos não-lineares para obter precisão e estabilidade nas soluções numéricas.
\end{abstract}

\section{Introdução}

São muitas as aplicações em que há a necessidade de se compreender os processos turbulentos nos fluidos. Por exemplo, o desprendimento de vórtices em pontes suspensas, a formação de saltos hidráulicos em canais abertos e películas sobre superfícies sólidas, o fluxo de sangue através do coração ou, ainda, a dispersão de poluentes na atmosfera terrestre. Em geral, os números de Reynolds envolvidos nesses problemas são elevados, ocasionando amplo intervalo entre as pequenas e as grandes escalas do escoamento. Nessas circunstâncias, a simulação a partir dos 
primeiros princípios do regime turbulento exige uma malha excessivamente fina e um passo de tempo bastante reduzido para que todas as escalas sejam adequadamente resolvidas. Complicações adicionais surgem quando se deseja estudar o comportamento da turbulência na presença de contornos rígidos e/ou livres, como é o caso de jatos em altas velocidades incidindo sobre superfícies sólidas ou o desprendimento de vórtices em obstáculos.

Para escoamentos incompressíveis, a grande maioria dos resultados na literatura são para o movimento de fluidos em regiões confinadas por paredes; poucos relatam experimentos numéricos de problemas com superfícies livres. Como decorrência, a análise dos métodos numéricos para resolver o estado turbulento associado a contornos livres e rígidos têm aparecido somente esporadicamente. Assim, o objetivo do presente trabalho é apresentar resultados de simulações numéricas, obtidas com o código GENSMAC [13], de escoamentos incompressíveis em regimes turbulentos e envolvendo contornos rígidos e/ou móveis. As simulações são realizadas utilizandose modelos de turbulência $\kappa-\varepsilon$ acoplados às equações de Navier-Stokes com média de Reynolds. Os termos não-lineares das equações de conservação são discretizados pelos esquemas HLPA ("Hybrid-Linear Parabolic Approximation") [17] e VONOS ("Variable-Order Non-Oscillatory Scheme") [14].

\section{Modelos de Turbulência $\kappa-\varepsilon$}

Em variáveis primitivas $(\mathbf{v}, p)$ e em forma conservativa, as equações de Navier-Stokes com média de Reynolds, para o caso incompressível, são dadas por:

$$
\begin{gathered}
\frac{\partial v_{i}}{\partial t}+\frac{\partial\left(v_{i} v_{j}\right)}{\partial x_{j}}=-\frac{\partial p}{\partial x_{i}}+\frac{\partial}{\partial x_{j}}\left(\nu \frac{\partial v_{i}}{\partial x_{j}}-\overline{v_{i}^{\prime} v_{j}^{\prime}}\right)+g_{i}, \quad i=1,2, \\
\frac{\partial v_{i}}{\partial x_{i}}=0
\end{gathered}
$$

em que $v_{i}$ e $v_{i}^{\prime}, i=1,2$, são, respectivamente, as componentes médias e flutuantes da velocidade nas direções $x_{i}, i=1,2, t$ é o tempo, $p$ é a pressão cinemática média (pressão dividida pela densidade), $g_{i}, i=1,2$, são as componentes do campo gravitacional, $\overline{v_{i}^{\prime} v_{j}^{\prime}}, i=1,2 ; j=1,2$, são as componentes do tensor de tensões de Reynolds, e $\nu$ é a viscosidade cinemática molecular. Nos modelos $\kappa-\varepsilon$, o tensor de Reynolds é relacionado ao tensor médio de deformações pela aproximação:

$$
-\overline{v_{i}^{\prime} v_{j}^{\prime}}=\nu_{t}\left(\frac{\partial v_{i}}{\partial x_{j}}+\frac{\partial v_{j}}{\partial x_{i}}\right)-\frac{2}{3} \kappa \delta_{i j}=\nu_{t} D_{i j}-\frac{2}{3} \kappa \delta_{i j},
$$

em que $\nu_{t}$ e $\kappa$ são, respectivamente, a viscosidade e a energia cinética turbulentas. As equações de transporte para $\kappa$ e sua dissipação $\varepsilon$ podem ser obtidas das equações (2.1) [6]:

$$
\begin{gathered}
\frac{\partial \kappa}{\partial t}+\frac{\partial\left(\kappa v_{j}\right)}{\partial x_{j}}=\frac{\partial}{\partial x_{j}}\left(\left(\nu+\nu_{t} / \sigma_{\kappa}\right) \frac{\partial \kappa}{\partial x_{j}}\right)+\nu_{t} D_{i j} \frac{\partial v_{i}}{\partial x_{j}}-\varepsilon \\
\frac{\partial \varepsilon}{\partial t}+\frac{\partial\left(\varepsilon v_{j}\right)}{\partial x_{j}}=\frac{\partial}{\partial x_{j}}\left(\left(\nu+\nu_{t} / \sigma_{\varepsilon}\right) \frac{\partial \varepsilon}{\partial x_{j}}\right)+\left(C_{1 \varepsilon} \nu_{t} D_{i j} \frac{\partial v_{i}}{\partial x_{j}}-C_{2 \varepsilon} \varepsilon\right) / T_{t}+E
\end{gathered}
$$


em que $C_{1 \varepsilon}=1.44, C_{2 \varepsilon}=1.92, \sigma_{\kappa}=1.0, \sigma_{\varepsilon}=1.3$ são constantes empíricas. A viscosidade turbulenta $\nu_{t}$, o tempo de escala $T_{t}$ e o termo-fonte $E$ assumem expressões diferentes, segundo o modelo $\kappa-\varepsilon$ escolhido. Neste trabalho, dois modelos particulares foram selecionados: o modelo clássico para altos números de Reynolds (HRe) [8], em que $\nu_{t}=C_{\mu} \kappa^{2} / \varepsilon, C_{\mu}=0.09, T_{t}=\kappa / \varepsilon$ e $E=0$; e o modelo para baixos números de Reynolds (LRe) [16], em que

$$
\nu_{t}=C_{\mu} f_{\mu} \kappa T_{t}, \quad T_{t}=\frac{\kappa}{\varepsilon}+C_{k}\left(\frac{\nu}{\varepsilon}\right)^{1 / 2}, \quad E=\nu \nu_{t} \frac{\partial}{\partial x_{k}}\left(\frac{\partial v_{i}}{\partial x_{j}}\right) \frac{\partial}{\partial x_{k}}\left(\frac{\partial v_{i}}{\partial x_{j}}\right)
$$

$f_{\mu}=\left(1-\operatorname{Exp}\left(-a_{1} R e_{z}-a_{3} R e_{z}^{3}-a_{5} R e_{z}^{5}\right)\right)^{1 / 2}, z$ a distância normal à fronteira rígida, $R e_{z}=\frac{z \kappa^{1 / 2}}{\nu}, a_{1}=1.5 \times 10^{-4}, a_{3}=5.0 \times 10^{-7}, a_{5}=1.0 \times 10^{-10}$, e $C_{k}=1$.

As equações (2.1)-(2.6) foram implementadas na forma adimensional, utilizandose as seguintes transformações:

$$
\begin{gathered}
v_{i}=v_{i}^{*} U, \quad x_{i}=x_{i}^{*} L, \quad t=t^{*} L / U, \quad p=p^{*} U^{2}, \quad g_{i}=g_{i}^{*}|g|, \\
\nu_{t}=\nu_{t}^{*} \nu, \quad \kappa=\kappa^{*} \nu U / L, \quad \varepsilon=\varepsilon^{*} \nu U^{2} / L^{2}
\end{gathered}
$$

em que $L$ e $U$ são, respectivamente, o comprimento e a velocidade característicos das grandes escalas, e $|g|$ é a magnitude da aceleração gravitacional.

\section{Condições Iniciais e de Fronteira}

Para se obter um problema de valor inicial e de fronteira bem posto é preciso especificar condições iniciais e de fronteira para as variáveis dependentes nos modelos $\kappa-\varepsilon$. Em geral, as condições iniciais para $\kappa$ e $\varepsilon$ são difíceis de serem especificadas, uma vez que elas devem estar em concordância com a física do problema. Desta forma, esse tipo de informação nem sempre está disponível em escoamentos envolvendo superfícies livres. Como estamos considerando um escoamento uniforme na entrada do domínio de solução, uma alternativa é prescrever os valores de $\kappa$ e $\varepsilon$ (e, portanto, $\nu_{t}$ ) em função de uma intensidade de turbulência, como é feito em diversos trabalhos na literatura, por exemplo [12]. Neste trabalho, definimos as variáveis turbulentas adimensionais em função de uma intensidade $I$ de turbulência e do número de Reynolds $(R e=L U / \nu)$ como $\kappa=I R e$ e $\varepsilon=(1 / 0.03)\left(R e^{-1} \kappa^{3}\right)^{1 / 2}$.

Nos escoamentos envolvendo superfícies livres, o tratamento das condições de fronteira associadas à essas interfaces tem impacto crucial na qualidade da simulação. Para se obter as condições fisicamente apropriadas, utilizamos o conceito de forças de superfície. Geralmente, a força de superfície é uma função da posição e da orientação da superfície em um dado ponto, e está associada ao vetor normal exterior unitário $\mathbf{n}=\left(n_{x}, n_{y}\right)$ naquele ponto. Assim, a tensão (força de superfície por unidade de área), é uma função $\tau_{i}=\tau_{i}\left(n_{i} ; x_{i}\right), i=1,2$, e é possível mostrar [11] que a dependência de $\tau_{i}$ sobre $n_{i}$ é dada por $\sigma \cdot \mathbf{n}=\tau$, em que $\sigma$ é o tensor 
efetivo de tensões viscosas definido como $\sigma_{i j}=\left(-p \delta_{i j}+\nu D_{i j}\right)+\left(-p_{t} \delta_{i j}+\nu_{t} D_{i j}\right)$, sendo $p_{t}$ a pressão turbulenta, e $\tau$ é a tensão na superfície. Nos problemas em que estamos interessados, o fluido está em contato com uma atmosfera inerte. Portanto, na superfície livre do fluido, a continuidade das tensões é imposta. Decompondo o vetor de tensões em componentes normais e tangenciais, obtemos as condições na fronteira livre [7]:

$$
n_{i}\left(\sigma_{i j} n_{j}\right)=0, \quad m_{i}\left(\sigma_{i j} n_{j}\right)=0,
$$

em que $\mathbf{m}=\left(m_{x}, m_{y}\right)$ é o vetor tangente à superfície livre. Substituindo o tensor de tensões $\sigma_{i j}$ nas equações (3.1) e utilizando as mudanças de variáveis (2.7) e (2.8), para adimensionalização, obtemos as formas das condições na interface:

$$
\begin{gathered}
p_{e}-\frac{2}{R_{e}}\left(1+\nu_{t}\right)\left[\frac{\partial u}{\partial x} n_{x}^{2}+\frac{\partial v}{\partial y} n_{y}^{2}+\left(\frac{\partial u}{\partial y}+\frac{\partial v}{\partial x}\right) n_{x} n_{y}\right]=0 \\
\frac{1}{R_{e}}\left(1+\nu_{t}\right)\left[2 \frac{\partial u}{\partial x} m_{x} n_{x}+2 \frac{\partial v}{\partial y} m_{y} n_{y}+\left(\frac{\partial u}{\partial y}+\frac{\partial v}{\partial x}\right)\left(m_{x} n_{y}+m_{y} n_{x}\right)\right]=0 .
\end{gathered}
$$

A partir da equação (3.2) determinamos a pressão efetiva $p_{e}$. Desde que $\nu_{t}>0$, (3.3) fornece o campo de velocidades. As equações (3.2) e (3.3) são aproximadas localmente por diferenças finitas, considerando-se as diferentes orientações da superfície livre [13]. As versões discretas dessas equações são obtidas aproximando-as em pontos específicos da superfície e exigindo-se conservação de massa. As condições de fronteira para $\kappa$ e $\varepsilon$ na superfície livre são as mais difíceis de serem especificadas devido à complexidade da dinâmica da turbulência próximo à interface, e até o presente momento esta questão permanece aberta. Por exemplo, não se sabe como a turbulência interage com a tensão superficial e, por consequência, é difícil estimar a distribuição de $\kappa$ sobre uma superfície irregular em movimento. Portanto, como uma primeira aproximação, assumimos que a superfície livre é localmente planar e que o movimento ao acaso do fluido não causa descontinuidades na fronteira. Formalmente, se $\phi=\kappa$ ou $\varepsilon$, então $\nabla \phi \cdot \mathbf{n}=0$.

As "condições de fronteira" em um contorno rígido para o modelo HRe são impostas utilizando-se as funções de parede $u^{*} U^{-1} \ln \left(A u^{*} U^{-1}\right)-a u=0$, $\kappa=C_{\mu}{ }^{-1 / 2} R_{e} u^{* 2}$ e $\varepsilon=R_{e} u^{* 3} / a z$ [8], em que $u^{*}$ é a "velocidade de atrito", $A=\operatorname{Re} \operatorname{Exp}(a C) z, C=5.45, a=0.418$, e $u$ é a magnitude da velocidade referente à distância $z$ da parede. No modelo LRe, as condições de contorno sobre fronteiras rígidas são obtidas pela imposição de que $\kappa=\nu_{t}=0$ nesses contornos. A variável $\varepsilon$ é, então, determinada a partir da equação de evolução da energia cinética $\kappa(2.4)$ e o resultado final é dado por $\varepsilon=\nu \frac{\partial}{\partial z}\left(\frac{\partial \kappa}{\partial z}\right)$. Na prática, a utilização dessa condição pode acarretar instabilidade numérica, uma vez que a segunda derivada não garante positividade de $\varepsilon$. Então, a seguinte condição, matematicamente equivalente [1], é utilizada na implementação $\varepsilon=2 R e^{-1}\left(\partial \kappa^{1 / 2} / \partial z\right)^{2}$.

Nos contornos de entrada ("inflow") e/ou saída ("outflow"), todas as variáveis dependentes são prescritas, utilizando-se as condições homogêneas de Neumann e/ou Dirichlet: para as velocidades médias, as condições são idênticas àquelas do caso laminar [13], isto é, na fronteira rígida aplica-se a condição de aderência completa ("no-slip"), e no contorno de simetria adota-se o deslizamento ("free-slip"); 
consistentemente com as condições iniciais, a variável $\kappa$ é prescrita na entrada do domínio de solução e seu valor é proporcional à componente relevante do campo de velocidades. Nesse caso, $\varepsilon$ é determinada pela relação $\varepsilon=(1 / 0.03)\left(R e^{-1} \kappa^{3}\right)^{1 / 2}$.

\section{Método Computacional e Discretizações}

As equações-modelo (2.1)-(2.6) são resolvidas explicitamente, de maneira análoga àquelas do código GENSMAC. Nas equações que modelam o movimento turbulento, os termos viscosos e os gradientes de pressão são aproximados utilizando-se diferenças centrais de segunda ordem, enquanto as derivadas temporais são discretizadas por diferenças avançadas. Diferentemente da maneira usual [12], todos os termos convectivos são discretizados por esquemas que satisfazem o critério CBC ("Convection Boundedness Criterium") [4],[9]. Em particular, os esquemas HLPA e VONOS foram escolhidos por apresentarem, em sua formulação, um bom compromisso entre estabilidade, precisão e simplicidade de implementação. Os detalhes da aplicação/implementação desses esquemas podem ser encontrados em [2] [3].

\section{Resultados Numéricos e Discussões}

Incrementado dos esquemas HLPA e VONOS e dos modelos HRe e LRe, o código GENSMAC simulou os problemas ilustrados na Figura 1:

MODELO I: - jato apresentando superfície livre, em que $D=6.0 \times 10^{-3} \mathrm{~m}$, $U=30 \mathrm{~ms}^{-1}, \quad \nu=1.0 \times 10^{-6} \mathrm{~m}^{2} \mathrm{~s}^{-1}, \quad R e=D U / \nu=1.8 \times 10^{5} \mathrm{e}$ $F r=U / \sqrt{g D}=1.23655 \times 10^{2}$;

MODELO II: - escoamento confinado sobre um obstáculo, em que $D=1.0 \times 10^{-2} \mathrm{~m}, U=2.5 \mathrm{~ms}^{-1}, \nu=1.0 \times 10^{-6} \mathrm{~m}^{2} \mathrm{~s}^{-1}$ e $R e=D U / \nu=2.5 \times 10^{4}$.

As malhas computacionais utilizadas nas simulações do modelo I foram $100 \times 50$ $(d x=d y=0.0005 m)$, que chamamos de malha GROSSA, e $200 \times 100$ $(d x=d y=0.00025 m)$ que chamamos de malha FINA. Para o modelo II, as malhas GROSSA e FINA foram, respectivamente, $120 \times 75(d x=d y=0.01 \mathrm{~m})$ e $240 \times 150(d x=d y=0.001 m)$. As Figuras 2-3 e 4-5 mostram, respectivamente, os resultados das simulações numéricas dos modelos I e II, utilizando-se ambas as malhas GROSSA e FINA e ambos os esquemas HLPA e VONOS.

Muito embora as condições de contorno na fronteira rígida sejam diferentes para os modelos HRe e LRe, os resultados numéricos nas duas malhas, para o jato com superfície livre, mostram-se semelhantes, como ilustrado pelas Figuras 2 e 3. Nesse mesmo problema, ao empregarmos uma malha mais fina nas simulações, isto é $400 \times 200(d x=d y=0.000125 m)$, verificamos que as soluções numéricas, obtidas com ambos os modelos HRe e LRe, convegiram para uma solução que está muito próxima daquela obtida com o modelo LRe, na malha FINA. Esse fato é uma forte indicação de que, no caso do MODELO I, a solução numérica é independente da malha. Após um certo tempo, o domínio de solução foi totalmente preenchido de fluido e a simulação foi encerrada com sucesso. 


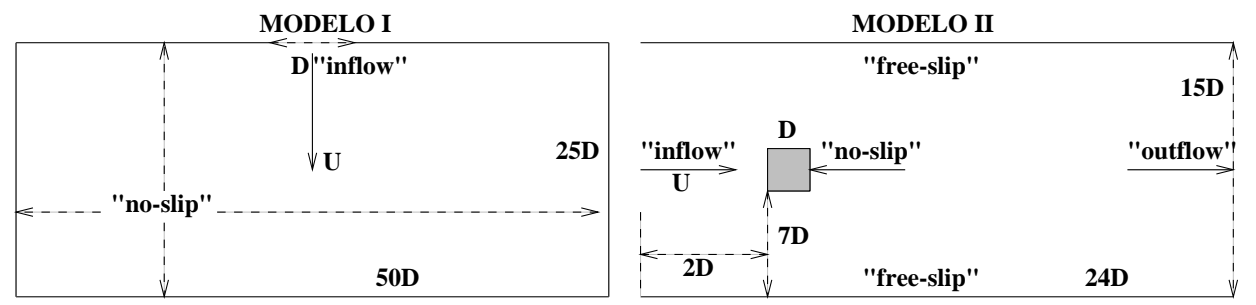

Figura 1: Geometrias para os escomentos estudados.
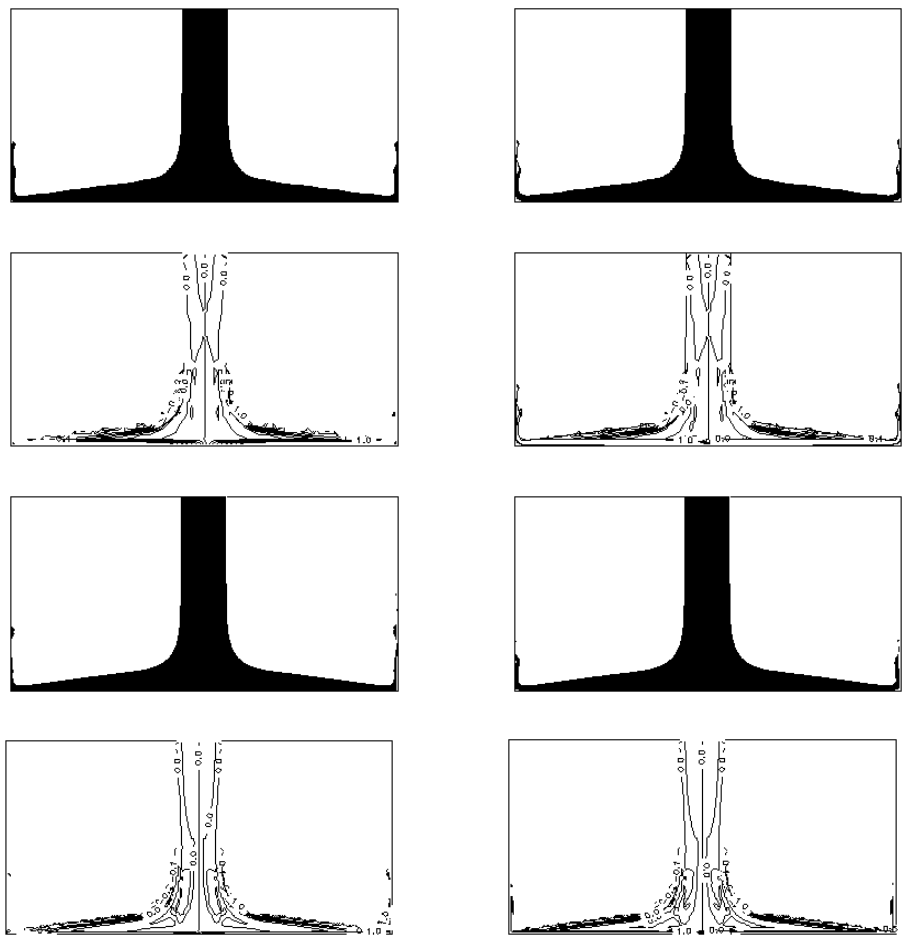

Figura 2: Superfície e campo de vorticidade, em $t=7.5$ (adimensional), de um jato sobre uma superfície rígida, utilizando-se os modelos LRe (à esquerda) e HRe (à direita). A primeira e segunda linhas de figuras correspondem à malha GROSSA, e a terceira e quarta à malha FINA. O esquema HLPA foi usado.

A despeito de o modelo II não apresentar superfície livre, foi difícil simular a esteira de vórtices atrás do obstáculo. Acreditamos que a separação da camada limite e a presença de quinas no obstáculo causaram as dificuldades. Nas Figuras 4 e 5, observa-se que a esteira, obtida com o modelo HRe nas malhas GROSSA e FINA e com os esquemas HLPA e VONOS, apresentou, aparentemente, um movimento organizado. Embora isto parece não ocorrer em situações práticas [5], os 
nossos resultados estão, qualitativamente, de acordo com a simulação apresentada em [10]. Nós atribuímos essas discrepâncias às funções de parede para as condições de contorno no obstáculo e à difusão numérica. Por outro lado, as simulações com o modelo LRe apresentou uma esteira de vórtices mais consistente com dados da literatura. Um refinamento da malha FINA foi também realizado nesse caso, e os resultados mostraram-se semelhantes aos obtidos naquela malha.
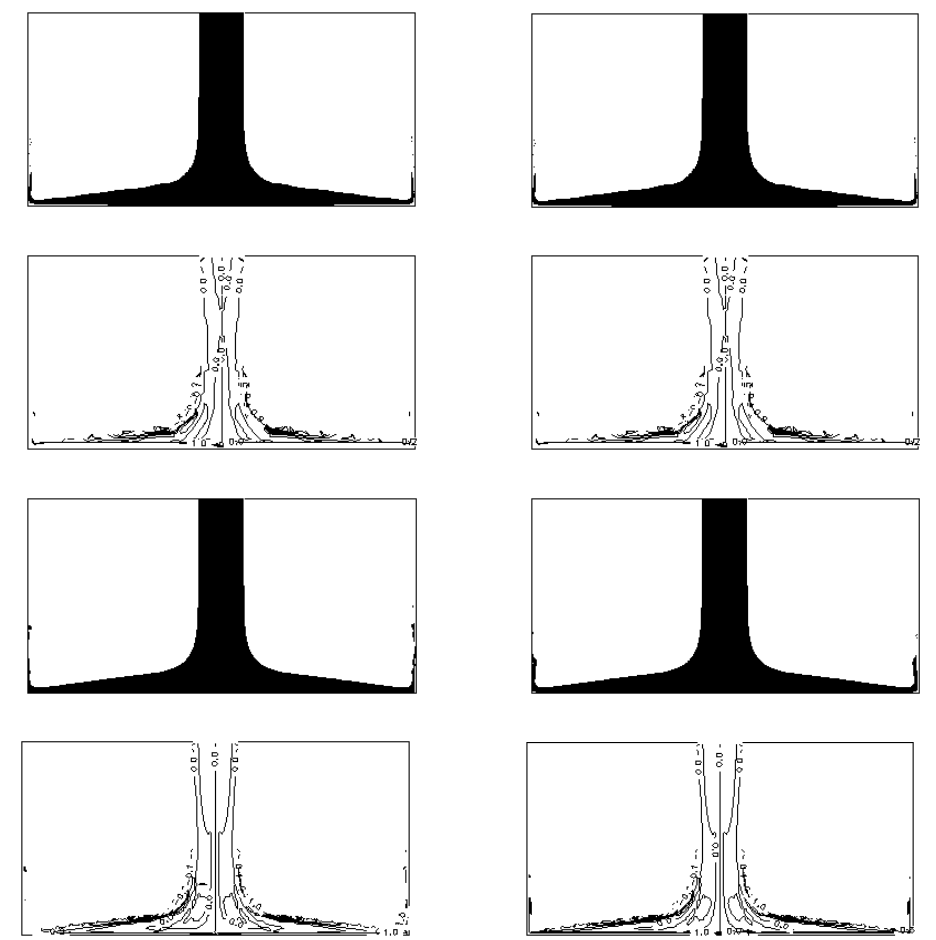

Figura 3: Superfície e campo de vorticidade, em $t=7.5$ (adimensional), de um jato sobre uma superfície rígida, utilizando-se os modelos LRe (à esquerda) e HRe (à direita). A primeira e segunda linhas de figuras correspondem à malha GROSSA, e a terceira e quarta à malha FINA. O esquema VONOS foi usado.

\section{Conclusões}

Duas versões dos modelos de turbulência $\kappa-\varepsilon$, e esquemas de convecção limitados e de segunda ordem, foram analisados e implementados no código GENSMAC para a simulação de escoamentos turbulentos envolvendo fronteiras livres e/ou rígidas. Dois problemas-teste foram considerados: um jato incidindo sobre superfícies sólidas e uma corrente em um canal contendo um obstáculo.

Os esquemas HLPA e VONOS mostraram ser pouco dissipativos por natureza, 

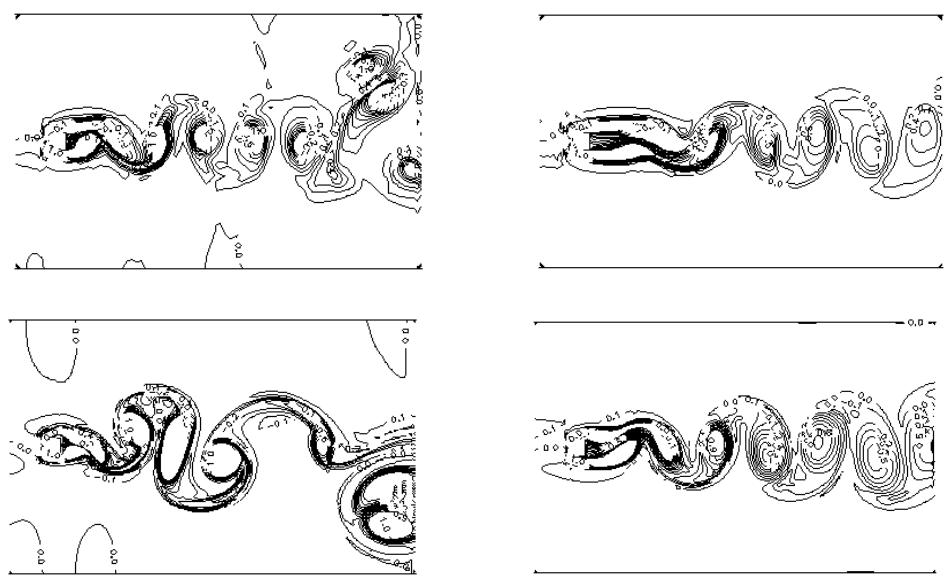

Figura 4: Campo de vorticidade, em $t=180$ (adimensional), de uma corrente confinada ao redor de um obstáculo, utilizando-se os modelos LRe (à esquema) e HRe (à direita). A primeira linha de figuras corresponde à malha GROSSA, e a segunda à malha FINA. O esquema HLPA foi utilizado para aproximar os termos convectivos.
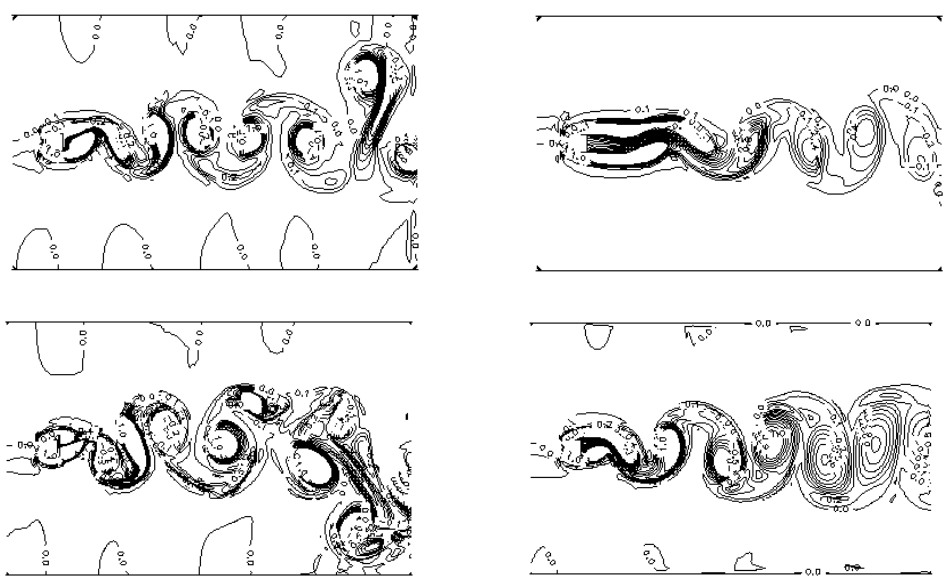

Figura 5: Campo de vorticidade, em $t=180$ (adimensional), de uma corrente confinada ao redor de um obstáculo, utilizando-se os modelos LRe (à esquema) e HRe (à direita). A primeira linha de figuras corresponde à malha GROSSA, e a segunda à malha FINA. O esquema VONOS foi utilizado para aproximar os termos convectivos.

e são bons canditatos para o tratamento dos termos não-lineares das equações di- 
ferenciais parciais que transportam propriedades físicas. Em particular, o esquema VONOS pareceu introduzir menos viscosidade artificial nos problemas considerados.

O presente estudo nos dá fortes indicações de que o código GENSMAC, adaptado com esquemas "upwind" de segunda ordem limitados e modelos de turbulência $\kappa-\varepsilon$, é, de fato, adequado para simular escoamentos em qualquer regime do número de Reynolds; muito embora a questão das condições do contorno para as fronteiras livre e rígida ser ainda um assunto de muita controvérsia.

Finalmente, como extensão do presente trabalho, pretendemos: (1) tratar o problema fundamental da precisão dos modelos em escoamentos envolvendo simetria radial [15]; (2) analisar a sensibilidade das soluções com respeito às variações das constantes empíricas; e (3) iniciar pesquisas na área de simulação em grandes escalas e até mesmo à direta.

Abstract. Good performance is the essential feature of any numerical algorithm for the simulation of turbulent fluid flow. This work presents numerical results of two incompressible turbulent flows with free surfaces and solid boundaries: a jet impinging onto a solid surface and vortex shedding behind an obstacle. These flows are solved in primitive variables formulation using the GENSMAC code. Two versions of the $\kappa-\varepsilon$ turbulence model were implemented: the classical and a low-Reynolds number model. The convective terms of the momentum and turbulence equations were approximated using bounded high-order upwind schemes. The results confirm the importance of adequate treatment of the convection terms to obtain stable and accurate numerical results.

\section{Referências}

[1] H.C. Chen e V.C. Patel, Near-wall turbulence models for complex flows including separation, AIAA Journal 26 (1988), 641-648.

[2] V.G. Ferreira, M.F. Tomé, N. Mangiavacchi, A.F. Castelo, J.A. Cuminato, A.O. Fortuna e S. Mckee, High order upwinding and the hydraulic jump, Int. J. Num. Meth. Fluids, (to appear).

[3] V.G. Ferreira, M.F. Tomé, N. Mangiavacchi, A.F. Castelo, J.A. Cuminato, A.O. Fortuna e S. Mckee, A comparative study of high-order upwind schemes on axisymmetric free surface flows, in "Third International Conference on Nonlinear Dynamics, Chaos, Control and Their Applications in Engineering Sciences" (J.M. Balthazar, et al., eds.), pp. 21-30, ABCM, Brazil, 2000.

[4] P.H. Gaskell e A.K. Lau, Curvature-compensated convective transport: SMART, a new boundedness-preserving transport algorithm, Int. J. Num. Meth. Fluids 8 (1988), 617-641.

[5] J.H. Gerrard, The mechanics of the formation region of vortices behind bluff bodies, J. Fluid Mech. 25 (1966), 401-413.

[6] F.H. Harlow e P.I. Nakayama, Turbulence transport equations, Physics of Fluids 10 (1967), 2323-2333. 
[7] L.D. Landau e E.M. Lifshitz, "Fluid Mechanics", 2ed. Pergamon Press, Oxford, 1980.

[8] B.E. Launder e D.B. Spalding, The numerical computation of turbulent flows, Int. J. Num. Meths. Fluids 15 (1974), 127-146.

[9] B.P. Leonard, Simple high-accuracy resolution program for convective modeling of discontinuities, Int. J. Num. Meth. Fluids 8 (1988), 1291-1318.

[10] B. Mohammadi e O. Pironneau, Unsteady separated turbulent flows computations with wall-laws and $\kappa-\varepsilon$ model, Comput. Methods Appl. Mech. Engrg. 148 (1997), 393-405.

[11] R.E. Nickell, R.I. Tanner e B. Caswell, The solution of viscous incompressible jet and free-surface flows using finite-element methods, J. Fluid Mech. 65 (1974), 189-206.

[12] B. Song, G.R. Liu e R.S. Amano, Application of a higher-order bounded numerical scheme to turbulent flows, Int. J. Num. Meth. Fluids 35 (2001), 371-394.

[13] M.F. Tomé e S. McKee, GENSMAC: A computational marker-and-cell method for free surface flows in general domains, J. Comput. Phys. 110 (1994), 171-186.

[14] A. Varonos e G. Bergeles, Development and assessment of a variable-order non-oscillatory scheme for convection term discretization, Int. J. Num. Meth. Fluids 26 (1998), 1-16.

[15] E.J. Watson, The radial spread of a liquid jet over a horizontal plane, J. Fluid Mech. 20 (1964), 481-499.

[16] Z. Yang e H. Shih, New time scale based $\kappa-\varepsilon$ model for near-wall turbulence, AIAA Journal 7 (1993), 1191-1198.

[17] J. Zhu, On the higher-order bounded discretization schemes for finite volume computations of incompressible flows, Comput. Meths. Appl. Mech. Engrg. 98 (1992), 345-360. 